\title{
The coherence of small island sea level with the wider ocean: a model study
}

\author{
Joanne Williams and Chris W. Hughes \\ National Oceanography Centre, 6 Brownlow St, Liverpool L3 5DA, UK \\ Correspondence to: Joanne Williams (joll@noc.ac.uk)
}

Received: 29 August 2012 - Published in Ocean Sci. Discuss.: 26 September 2012

Revised: 17 January 2013 - Accepted: 25 January 2013 - Published: 14 February 2013

\begin{abstract}
Studies comparing tide gauge measurements with sea level from nearby satellite altimetry have shown good agreement for some islands and poor agreement for others, though no explanation has been offered. Using the $1 / 12^{\circ}$ OCCAM ocean model, we investigate the relationship between sea level at small, open-ocean islands and offshore sea level. For every such island or seamount in the model, we compare the shallow-water sea level with the steric and bottom pressure variability in a neighbouring ring of deep water. We find a latitude-dependent range of frequencies for which off-shore sea level is poorly correlated with island sea level. This poor coherence occurs in a spectral region for which steric signals dominate, but are unable to propagate as baroclinic Rossby waves. This mode of decoupling does not arise because of islands bathymetry, as the same decoupling is seen between deep ocean points and surrounding rings.
\end{abstract}

\section{Introduction}

Tide gauges are necessarily situated on the coast, and in using tide-gauge data to draw inferences about the deeper ocean some assumptions must be made. Satellite altimetry, by contrast, has until recently performed poorly at the coast (Vignudelli et al., 2011). How does sea level at islands relate to that offshore? This question matters to island communities, but also because island tide gauges play a particularly important role in satellite altimetry calibration (Leuliette et al., 2004). It may also be significant for reconstructing global sea levels from historic tide gauge records.

The question has been addressed to some extent by Vinogradov and Ponte (2011), who compared tide gauge records to nearby sea-surface height derived from TOPEX/Poseidon altimetry. "Nearby" here refers to altimetry from within $180 \mathrm{~km}$, also usually from water more than $200 \mathrm{~m}$ deep. They found the percentage of variance in near-gauge altimetry data explained by the gauge is high $(>80 \%)$ for most Pacific islands and Indian Ocean sites, but low on North Atlantic and Pacific coasts. Furthermore, it is also very low $(<10 \%)$ at several sites in the eastern South Pacific - see Fig. 6 in their paper. They also hint at improved correlations when time series are averaged over longer periods -2 and $3 \mathrm{yr}$.

There are three ways in which coastal sea level can be decoupled from nearby deep water variability. First, there can be additional variability in shallow water, resulting from local effects of wind stress and atmospheric pressure. Second, deep water signals are often primarily steric (the result of changes in the depth-integrated density). Being a depth integral, steric signals necessarily become small in shallow water, so an offshore steric signal must be converted into a bottom-pressure signal if the associated sea-level change is to be communicated to the coast. This is what would happen in the absence of horizontal pressure gradients, but it is also possible for interactions between density and bathymetry to induce currents, and associated pressure gradients, which act to insulate the coast from offshore sea level changes. Third, it may simply be the case that the offshore variability does not propagate in an organized manner, and therefore it is not strongly correlated with any nearby variability, coastal or otherwise.

The first two of these mechanisms appear to be operating on some continental coasts, as illustrated in spectra of sea level variability in the range of periods of 2-24 weeks by Hughes and Williams (2010). These show several regions where variability in shallow water is dominated by highfrequency content, and in deeper water by low frequencies, 
with the two separated by low variability along the shelf edge. Thus, the deep water variability does not penetrate onto the shelf, and there is additional variability on the shelf with a spectrum representative of wind-driven processes.

Bingham and Hughes (2012) looked at continental coasts in the OCCAM $1 / 12^{\circ}$ model, reconstructing coastal sea level using various approximations. "Approximation A" in their paper was that sea-surface height is spatially constant near the coast, $\nabla p_{0}=0$, so the tide gauge can be approximated by steric height at a nearby point. This only does well near the Equator, with performance dropping poleward of about $20^{\circ}$, particularly on the western ocean boundaries. "Approximation C" in their paper was that the horizontal gradient of pressure anomaly at the bottom is zero; i.e. there is no geostrophic velocity at the bottom, $(\nabla p)_{\mathrm{b}}=0$. This is better than "Approximation A" for estimating the steric effect on tide gauges away from the Equator on eastern boundaries, and much better than A on western boundaries where there is a strong boundary current (though still worse than on eastern boundaries). These results show how boundary currents can significantly disrupt the link between coastal and deep ocean sea level, even on interannual time scales. Vinogradov and Ponte (2011) observed this in the Kuroshio off the coast of Japan, reporting that although "the tide gauge variability tends to be weak but relatively coherent along the coast, the [altimetry] series vary considerably".

For small islands, we might expect both the wind-stress and alongshore current mechanisms to be less effective. The closed, short bathymetric contours around an island may present less of a barrier to signal propagation, and the shallow water area over which wind stress acts will be smaller, producing less additional local variability. Nonetheless, in this model study we do see significant decoupling for many islands, and the much greater data availability in the model compared with the limited number of tide gauge records available in the real world allows us to investigate the spatial and spectral dependence of this decoupling in more detail, and also to extend the investigation to the open ocean. We find evidence for the importance of the third decoupling mechanism, which does not depend on the reference point being an island, or in shallow water, but operates at all open ocean points.

\section{Methods}

For this paper sea-surface height, steric height and bottom pressures are calculated from run 401 of the OCCAM $1 / 12^{\circ}$ model described by Marsh et al. (2009), sampled as 5-day means over the years 1988-2004. We will consider variability of inverse-barometer-corrected sea level $h$ to be the sum of variation in terms $p$ and $\phi$ associated with bottom pressure $p_{\mathrm{b}}$ and steric variations respectively, where $h=p+\phi=\frac{p_{\mathrm{b}}}{\rho_{0} g}-\frac{1}{\rho_{0} g} \int_{-H}^{0} \rho g \mathrm{~d} z$.

$\rho$ is density (with $\rho_{0}$ the model's Boussinesq reference density), $g$ the acceleration due to gravity, $z$ the vertical coordinate and $H$ ocean depth.

In this paper we define a mid-ocean "island" as a contiguous area of ocean shallower than $300 \mathrm{~m}$ and smaller than $25000 \mathrm{~km}^{2}$, which lies outside the contiguous region of continental seas to a depth of $2000 \mathrm{~m}$. Thus the Philippines are excluded for being "continental" and New Zealand for being too large, but Fiji is included. There is no requirement for there to actually be any land, so some small seamounts are included. With this generous definition we find islands in much of the ocean, though there are few in the deep east Pacific (the distribution can be seen in later figures).

For each island we compare sea-surface height $(h)$ averaged over this onshore-area with $h$ and its steric $(\phi)$ and bottom pressure $(p)$ components, averaged over a "ring" around the island, defined as all points deeper than $3000 \mathrm{~m}$ and closer than $2.5^{\circ}$ latitude and longitude ( $\sim 200 \mathrm{~km}$ at mid-latitudes) to the island centre (see Fig. 1).

To establish whether any effects are due to the island depth or simply distance, we also define "control islands" as $0.75^{\circ} \times 0.75^{\circ}$ squares of water over $3000 \mathrm{~m}$ deep and compare sea level there to the surrounding squares between widths $0.75^{\circ}$ and $2.5^{\circ}$ (intermediate ring) and between widths $2.5^{\circ}$ and $5^{\circ}$ (distant ring).

\section{Results}

\subsection{Global mean spectra}

Firstly, we compare the spectra of sea-level signals averaged over all the islands with those for deep oceans and continental coasts. The deep water $\phi$ variability (heavy and light red, Fig. 2) has a spectrum approximately proportional to $\sigma^{-1 / 2}$, steepening beyond $\sigma^{-2}$ at frequencies $(\sigma)$ greater than 3 cycles $\mathrm{yr}^{-1}$. Steric variability has much greater power than bottom pressure $p$ (heavy and light blue) in deep water, which has a more gently sloping spectrum. The near-island $p$ spectrum is a little less energetic than that for the deep ocean as a whole but very similar in shape. The $h$ signal at the islands (heavy black) has a spectrum similar in character but reduced in power compared to the deep $\phi$, suggesting an influence of nearby steric variability on island sea level, but a degree of decoupling resulting in reduced power. The island $h$ spectrum is very close to the continental coastal $h$ (light black) between about 4 months and a year, but for shorter periods the continental signal does not steepen so much and is more energetic. This corresponds to the "blue" high-frequency spectra seen on the continental coasts in the 

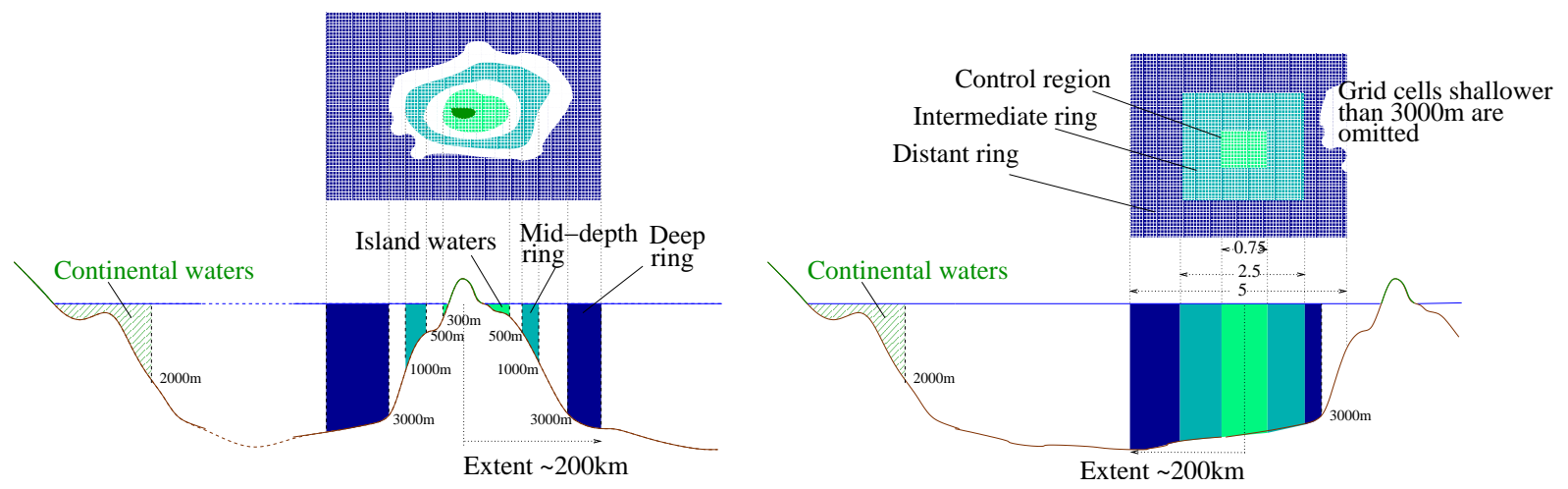

Fig. 1. Illustration of island waters and surrounding rings, and control regions.

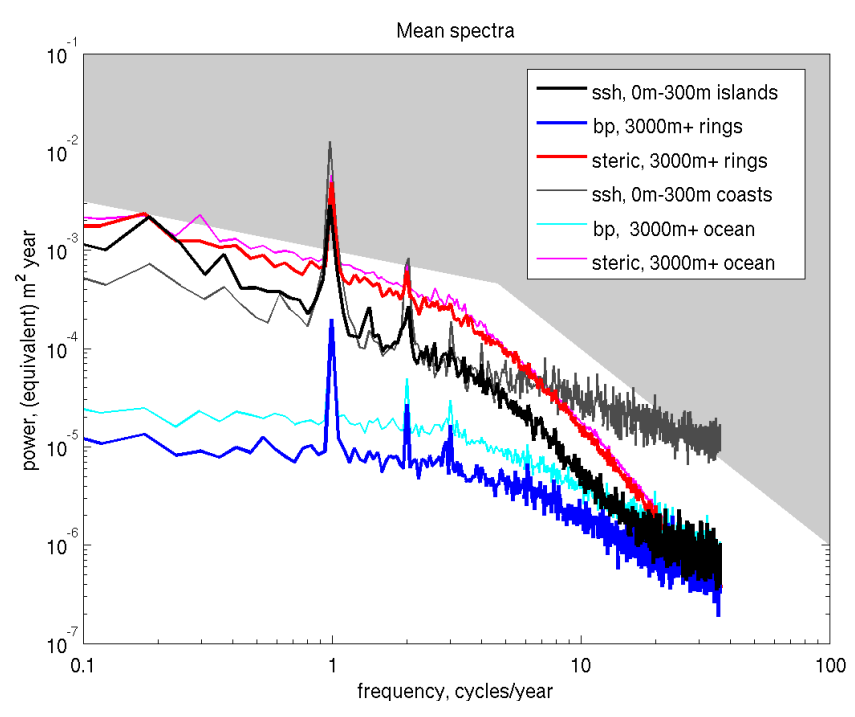

Fig. 2. Mean spectra of shallow-water sea-surface height $(h)$ (black/grey), and deep-water steric $(\phi)$ (red) or bottom pressure ( $p$ ) (blue) contributions to sea-surface height. Heavy curves are for spectra averaged over islands $(h)$ or surrounding deep rings $(\phi, p)$. Lighter curves are for continental shelves $(h)$ or deep water open ocean $(\phi, p)$. Polar regions are excluded. Background shading indicates power proportional to $\sigma^{-1 / 2}$ and $\sigma^{-2}$.

altimetry spectral maps of Hughes and Williams (2010). The greater high-frequency energy in coastal shallow water is due, in part at least, to the greater effect on sea level of wind over shallow water than over deep water. The rapid drop-off of the island sea level spectrum at these high frequencies is consistent with this process being a much less important source of decoupling at islands than on broad continental shelves, though we should note that the limitations of model resolution may lead to an underestimate of the energy in small-scale, local processes. The island sea level spectrum also approaches the near-island steric spectrum at the longest timescales, unlike the continental coastal sea level spectrum, perhaps suggesting that the decoupling decreases at the longest time scales.
The shapes of the spectra suggest that nearby steric-height signals do directly influence coastal island sea level, but that deep bottom pressure signals become the dominant influence at high frequency. But all these interpretations remain tentative when based on globally averaged spectra. A more detailed analysis is needed in order to understand the processes.

\subsection{Relationship between island and off-shore sea level}

\subsubsection{For islands}

To investigate geographically varying effects, we plot in Fig. 3a the percentage of variance of the island $h$ signal explained by $h$ in the surrounding ring of deep water. A latitude dependence emerges, with much higher variance explained at the Equator, dropping with latitude, and rising again in the Southern Ocean. A similar plot (not shown) for $p$ at the island explained by $h$ offshore is nearly identical to this, and a plot of $h$ explained by $\phi$ offshore is also very alike, with changes mainly in the Southern Ocean. Less than $5 \%$ of overall island variability is explained by the offshore $p$, except in the Southern Ocean where it rises to about $50 \%$.

What is the timescale upon which the offshore $\phi$ signal can approximate the island $h$ ? If we filter each signal to pass periods $<6$ months before calculating the percentage of variance (not shown), we find that at the shorter time scales the island $h$ variance explained by the offshore $\phi$ drops from a maximum of about $90 \%$ at the Equator to a maximum of $80 \%$. The latitude dependence remains, and polewards of $20^{\circ}$ latitude there are few islands for which the off-shore $\phi$ alone explains more than about $25 \%$ of the variance of the island $h$ signal. Offshore $\phi$ does almost as well as offshore $h$ except in the Southern Ocean where it is the offshore $p$ that explains the island $h$ at these timescales, with almost no contribution from $\phi$ (see Fig. 3b). There are not enough islands at equivalent northern latitudes to say whether this behaviour is limited to the Southern Hemisphere.

If we instead filter each signal to pass $>18$ months (Fig. 3c), we find that the variance explained by offshore $h$ improves slightly. A latitude dependence remains, with most 
(a) ssh at island, ssh in deep water nearby

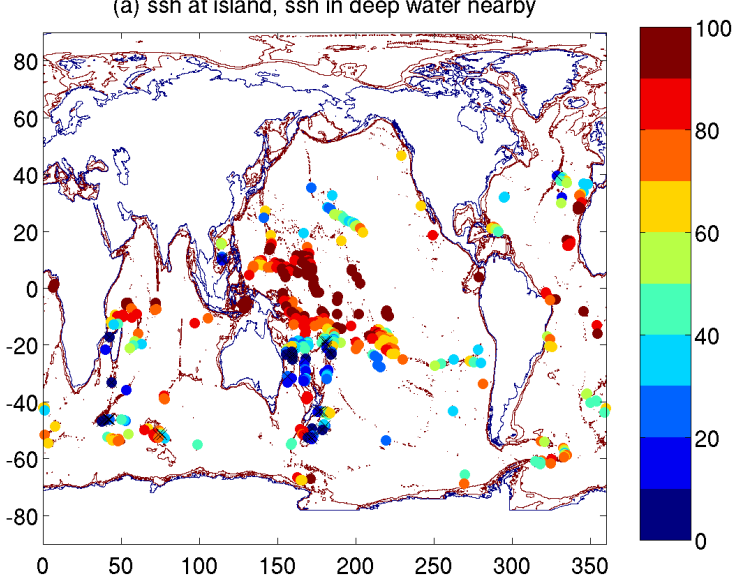

(b) ssh at island, bp in deep water nearby, 0-6 months

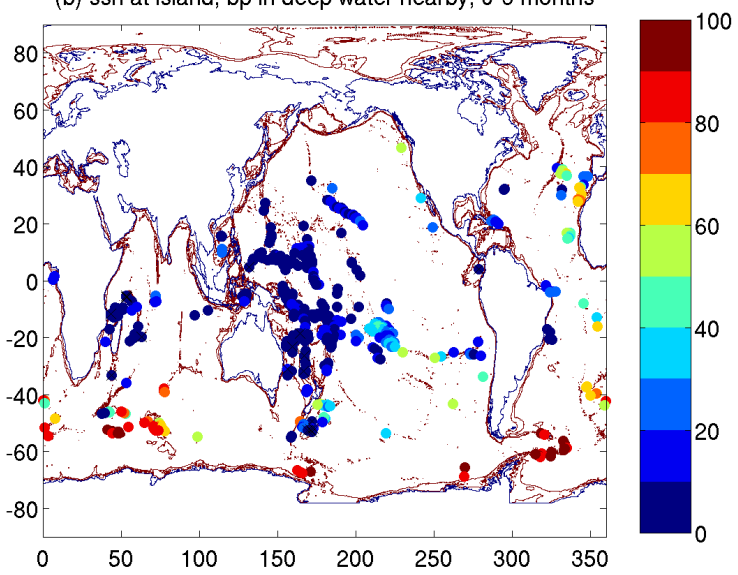

(c) ssh at island, ssh in deep water nearby, 18+ months

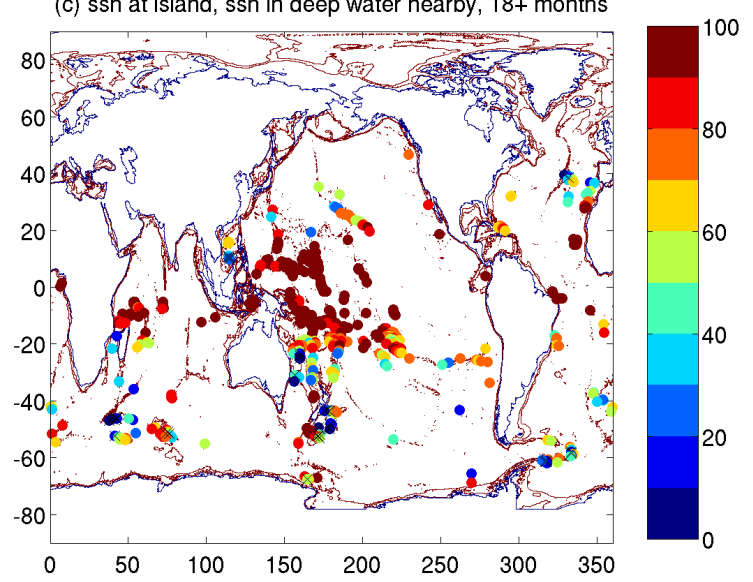

(d) ssh at island, ssh nearby

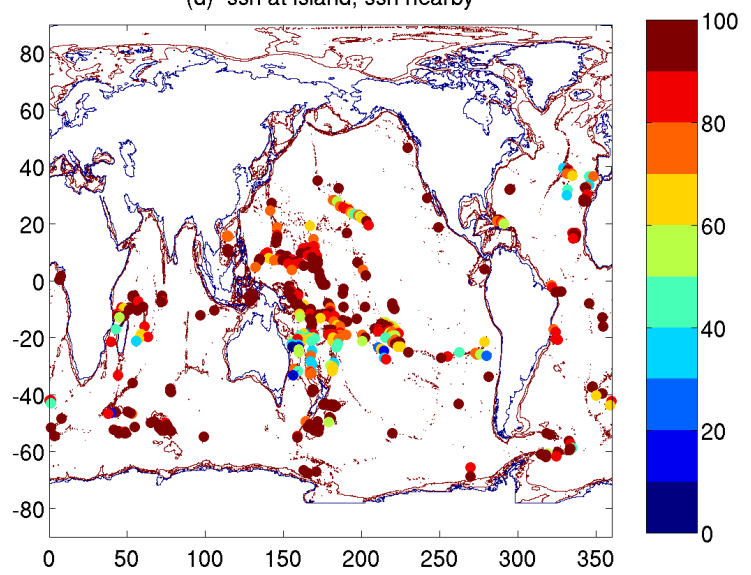

Fig. 3. Percentage of variance of $h$ at island explained by $h(\mathbf{a}, \mathbf{c}, \mathbf{d})$ or $p(\mathbf{b})$ in a neighbouring ring of water $3000 \mathrm{~m}$ deep (a-c) or $500-1000 \mathrm{~m}$ (d). (b) and (c) have 0-6 month high-pass and 18+ month low-pass filters applied respectively; (a) and (d) are unfiltered.

islands between $\pm 20^{\circ} \mathrm{N}$ having over $70 \%$ of variance explained by off-shore $\phi$ (not shown, similar to Fig. 3c), and islands between $40-20^{\circ} \mathrm{S}$ around $30-50 \%$. At these longer periods, offshore $p$ (not shown) makes very little contribution to the observed coherence anywhere.

\subsubsection{For "flat-bottomed control" regions}

The coherence (or lack of it) between island and off-shore sea level may be due to the bathymetry, or simply the distance between the island and deeper waters. The coherence between the distant sea level and that at the "control island" displays the same spatial dependence as for the island analysis (Fig. 4). In fact, Fig. 3a could almost be a subsampled version of Fig. 4.

The spatial variability in Fig. 4 is not a function of latitude alone, but includes some longitude dependence. Regions of high eddy energy, such as the Kuroshio, the Gulf Stream, the East Australia Current, the Agulhas retroflection and the Antarctic Circumpolar Current, all show reduced coupling. We will return to this below.

\subsection{Correlation by timescale}

To investigate the correlation by timescale in more detail, we calculate the magnitude squared coherence estimate, $\gamma_{x y}$, using the Matlab function mscohere. This is defined as

$\gamma_{x y}(\sigma)=\frac{\left|S_{x y}(\sigma)\right|^{2}}{S_{x x}(\sigma) S_{y y}(\sigma)}$

where $S_{x y}$ is the cross power spectral density; thus $\gamma_{x y}(\sigma)$ has values between 0 and 1 and indicates how well $x$, the signal at the island, corresponds to $y$, the signal in deep water, at each frequency (Emery and Thomson, 2001). $x$ and $y$ are chosen from $h, \phi$ and $p$.

Figure 5 shows $\gamma_{h h}, \gamma_{h \phi}, \gamma_{h p}$, the magnitude squared coherence of sea-surface height at islands and signals in deep water, averaged over four latitude bands. At the annual frequency, there is a sharp peak in $\gamma_{h \phi}$, especially at high latitudes. Otherwise, equatorwards of $35^{\circ} \gamma_{h \phi}$ generally decreases with higher frequencies, and $\gamma_{h p}$ increases with higher frequencies. $\gamma_{h \phi}$ is highest at the Equator for all frequencies, and at high frequencies $\gamma_{h p}$ appears to 


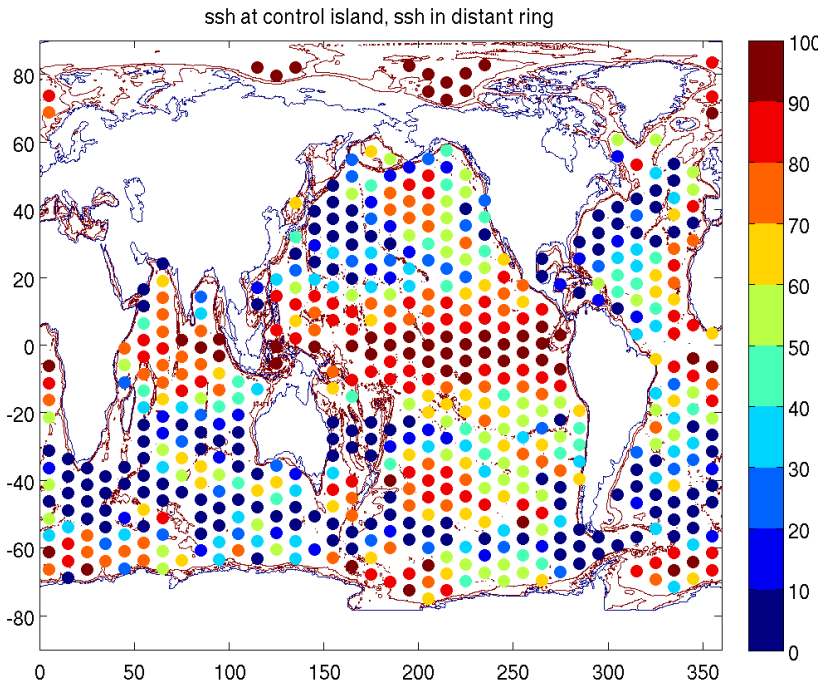

Fig. 4. Percentage of variance of $h$ at "control island" explained by $h$ in a $5^{\circ}$ diameter ring.

steadily increase with distance from the Equator. $\gamma_{h h} \approx$ $\max \left(\gamma_{h \phi}, \gamma_{h p}\right)$, with a dip in coherence at the transition between steric height and bottom pressure, which occurs over periods of between one month and one year according to latitude.

\subsection{Correlation by timescale and latitude}

This latitude dependence bears further investigation, and the three panels of Fig. 6 show $\gamma_{h h}, \gamma_{h \phi}$ and $\gamma_{h p}$ respectively, for every island by latitude and frequency. In Fig. 6a there is a high coherence for low frequencies (left) where the steric part of the offshore signal corresponds well to island $h$, and for high frequencies (right) where the bottom pressure part of the offshore signal corresponds well to $h$, but a zone of low coherence for intermediate frequencies.

The Rossby frequency, $\sigma_{\max }=\beta R_{0} / 4 \pi$, is the maximum frequency at which baroclinic Rossby waves can exist, based on the linear dispersion relation and the first baroclinic Rossby radius $R_{0}$, taken from Chelton et al. (1998). The zonal average (in water deeper than $3000 \mathrm{~m}$ ) Rossby frequency is marked in Fig. 6 as a yellow line and corresponds to periods ranging from about 4 weeks at $\pm 5^{\circ}$ to longer than a year at high latitudes. Above the maximum Rossby frequency, baroclinic Rossby waves are not possible, meaning that the only propagation mechanism for subinertial variability is advective.

Once again, $\gamma_{h h}$ in Fig. 6 panel (a) is close to the larger of $\gamma_{h \phi}$ and $\gamma_{h p}$ in panels (b) and (c). The transition region between $\gamma_{h \phi}$ and $\gamma_{h p}$ is emphasized by black bars. These show, averaged over islands in bands of $5^{\circ}$ latitude, the range between the highest frequency (shortest period) for which $\gamma_{h \phi}>\gamma_{h p}$ and the lowest frequency (longest period) for which $\gamma_{h \phi}<\gamma_{h p}$. All of these bars are to the right of the
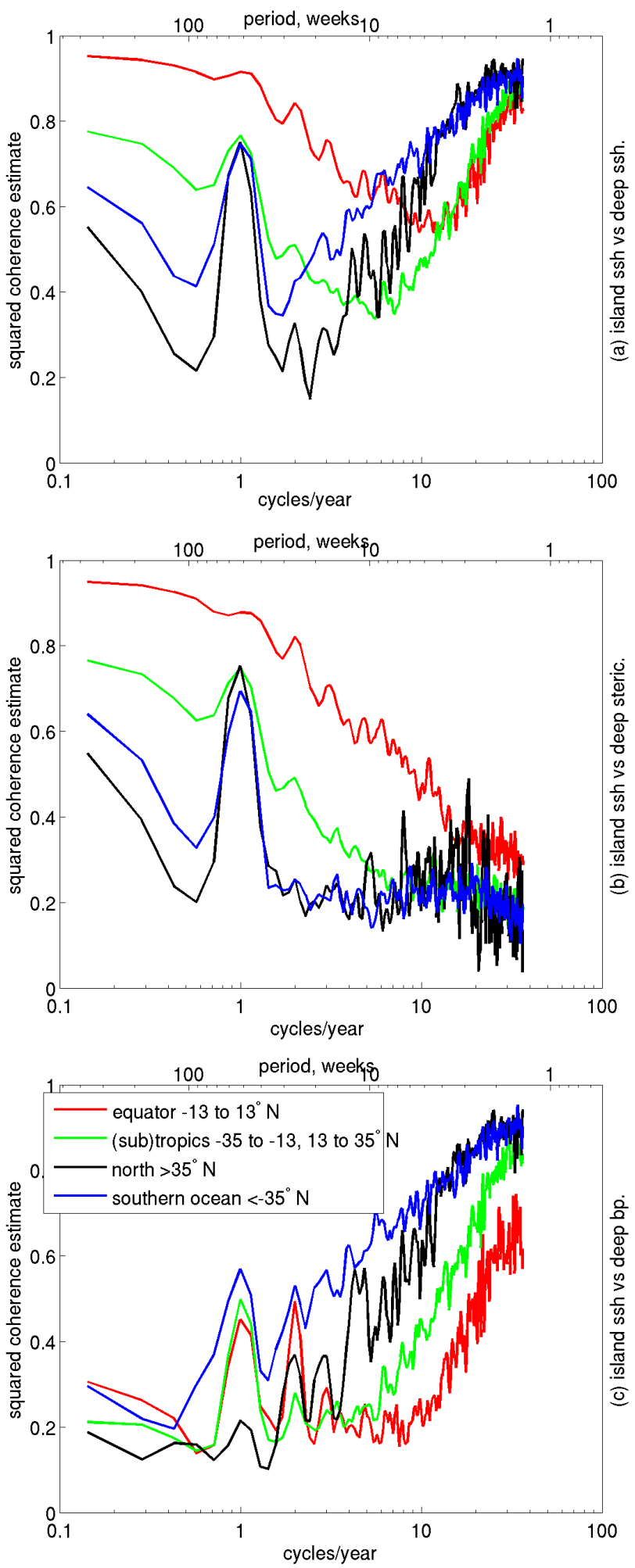

Fig. 5. Magnitude squared coherence estimate by frequency for sea-surface height at island and (a) sea-surface height $(h)$, (b) steric height $(\phi)$, and (c) bottom pressure $(p)$, in deep water nearby, averaged over latitude bands. 

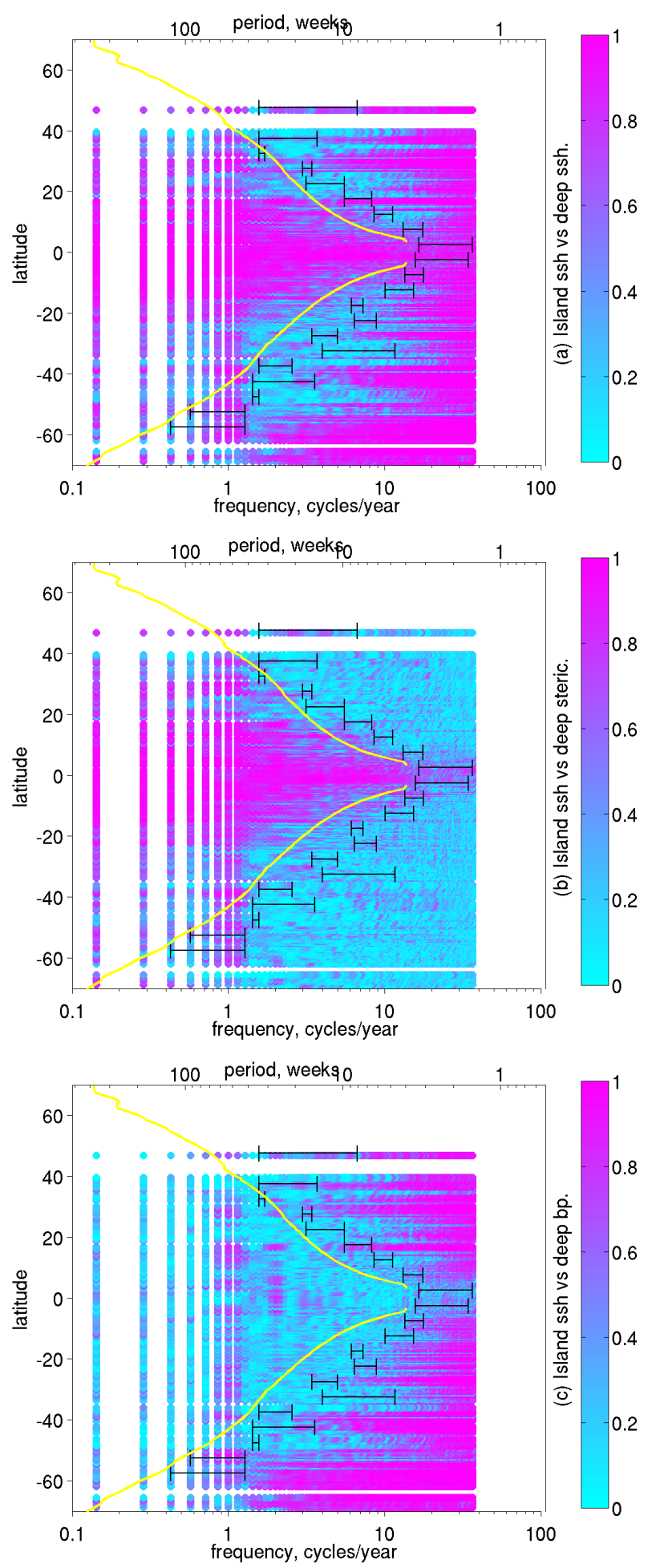

Fig. 6. Magnitude squared coherence of sea-surface height at island and (top to bottom) (a) sea-surface height (h), (b) steric height $(\phi)$, and (c) bottom pressure $(p)$, in deep water nearby, for every island by latitude and frequency. Pink dots indicate a high coherence and blue low. Black bars emphasize the transition region between dominance of $\gamma_{h \phi}$ and $\gamma_{h p}$. Linear baroclinic Rossby waves are possible only to the left of the yellow line. yellow line; thus at all latitudes the island $h$ is explained more by off-shore $\phi$ than off-shore $p$ for all frequencies lower than the Rossby wave frequency.

These transitions are well to the right of the low-coherence zone in Fig. 6a. So in the low-coherence zone the signal is primarily steric off-shore, but this is not sufficient for offshore $\phi$ to translate into an island $h$ signal. Only at timescales long enough for baroclinic Rossby waves does the off-shore steric height correlate well with the island sea-surface height.

Very similar behaviour is observed for coherence between sea level at our chosen deep water control regions and at a distant "ring" around them (Fig. 7, left-hand panels). This again supports the case that the primary mechanism responsible for variations in coherence is related to distance, and not to the presence of a shallow region around the islands.

\subsection{Intermediate depths and distances}

Bingham and Hughes (2012) found that although coastal sea level could be reconstructed using steric height at deep ocean $(3000 \mathrm{~m})$ sites near to tide gauges in the equatorial band, in most cases the steric correction could be improved by calculating steric height closer to the shore, in the depth range $500-1000 \mathrm{~m}$. We also find that, particularly at the shorter timescales and higher latitudes (excluding the Southern Ocean), the mid-depth $\phi$ does much better at explaining the island $h$, as would be expected as it is likely to be closer to the island. Figure $3 \mathrm{~d}$ shows the variance of island $h$ explained by $h$ in a ring defined as all points with depth $500-1000 \mathrm{~m}$ and closer than $2.5^{\circ}$ latitude and longitude to the island centre. The latitude dependence still exists, though it is weaker than in Fig. 3a, and most islands between $35^{\circ} \mathrm{S}$ and $13^{\circ} \mathrm{S}$ have around $40-80 \%$ of island $h$ variance explained by offshore $\phi$ (mid-depth ring) compared to 10-50\% (deep ring).

In our control region case, we can illustrate the effect of distance as a function of frequency and latitude by comparing the results using the standard distant ring with those from an intermediate ring (see Fig. 1). The resulting pattern of coherence (Fig. 7 right-hand panels), is very similar to that obtained using the more distant ring, (left-hand panels), though coherence is generally higher, as expected, making the dip in sea-level coherence less pronounced.

\subsection{Admittance}

We have also plotted (not shown) the admittance or transfer function for the islands and rings as defined above. This is defined by

$T_{x y}(\sigma)=\frac{S_{x y}(\sigma)}{S_{x x}(\sigma)}$,

where $S_{x y}$ is the cross power spectral density, and is calculated using the Matlab function tfestimate. Since admittance (unlike coherence) is not normalised by the power spectral density of the second signal, a high value requires the two 

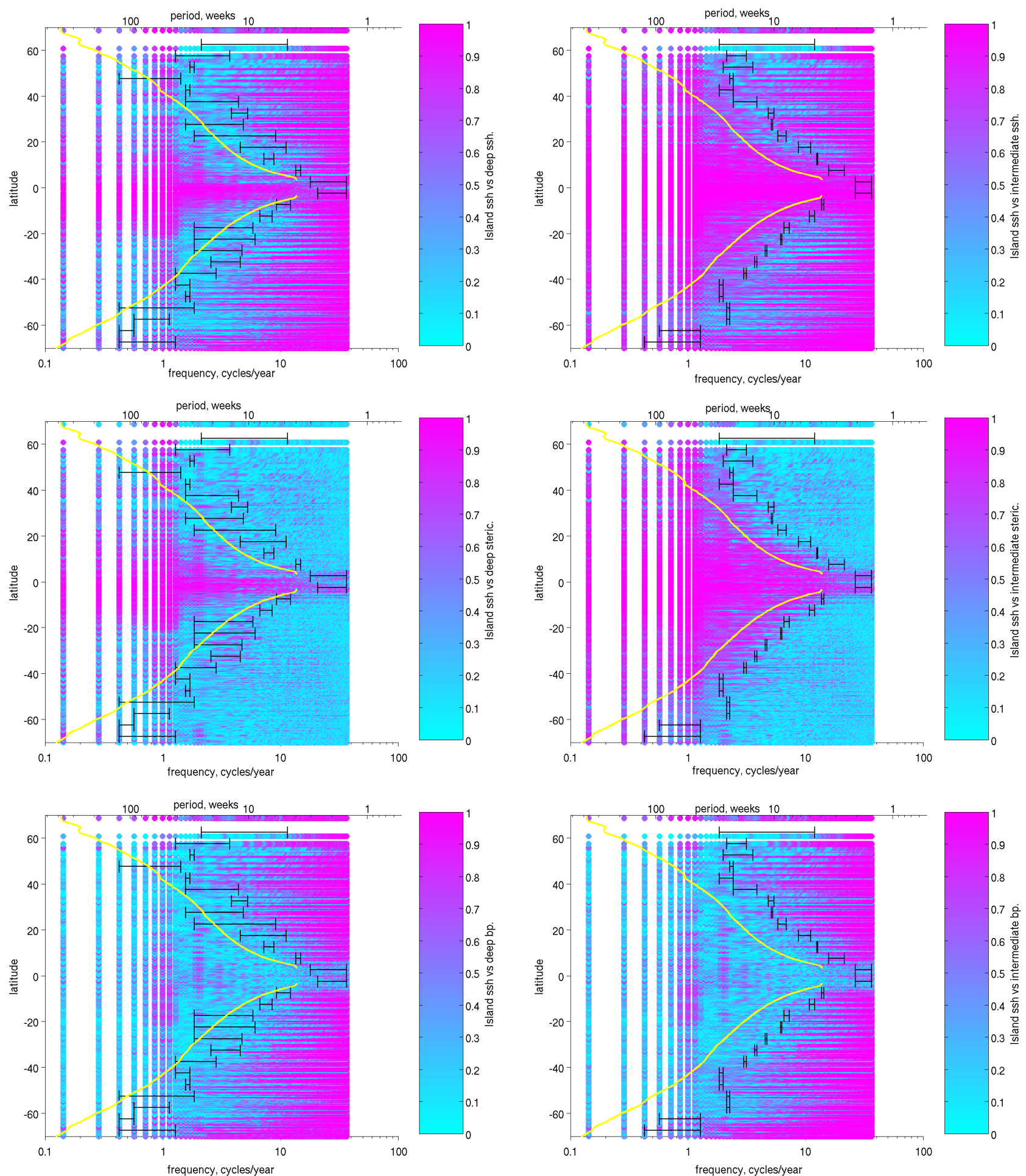

Fig. 7. Magnitude squared coherence of sea-surface height at "control island" and (panels from top to bottom) sea-surface height ( $h$ ), steric height $(\phi)$, and bottom pressure $(p)$, in surrounding ring. Left-hand panels are for distant ring, right-hand panels intermediate rings. Coherence is plotted for every island by latitude and frequency; pink dots indicate a high coherence and blue low. Black bars emphasize the transition region between $\gamma_{h \phi}$ and $\gamma_{h p}$. Linear baroclinic Rossby waves are possible only to the left of the yellow line. 

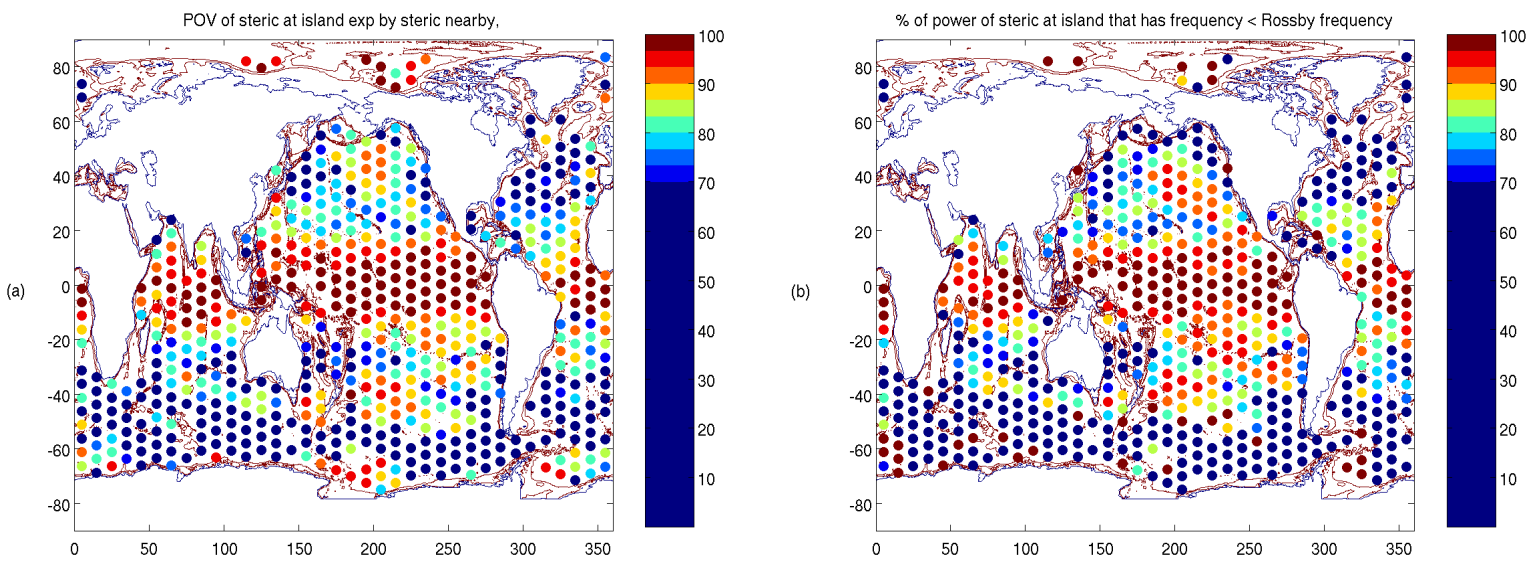

Fig. 8. (a) Percentage of variance of $\phi$ at "control island" explained by $\phi$ in a $2.5^{\circ}$ neighbouring ring. (b) Percentage of the power spectrum in $\phi$ at the "control island" that has frequency lower than the Rossby frequency at that location. Note change of scale from earlier maps.

signals to be of the same scale as well as varying together. This gives information about the ratios of the signals as well, but in fact in this case admittance looks very similar to coherence. The only exception is at the annual period, for which the admittance of island sea level to deep bottom pressure is very small despite coherence of up to 0.5 . This is simply a reflection of the fact that annual cycles are present in both steric and bottom pressure variability, but the steric signal usually has the greater amplitude. Outside the annual period band, the similarity between admittance and squared coherence means that Figs. 5, 6, and 7 give a good indication that the sea-level signal resulting from these coherent components is of the same amplitude as that observed at the islands, and not just well correlated.

\subsection{Spectral shape of steric signal}

The pattern in Fig. 4 is not purely a function of latitude. Points in the most energetic regions seem to have low $h$ variance explained by their neighbouring rings, tending to give lower values in the west of basins than in the east.

If we make the assumption that coherence from the steric variability only results from that part which falls to the left of the baroclinic Rossby wave cut-off frequency line (yellow lines in Figs. 5 and 6), then the percentage of variance explained will be the percentage of variance which lies to the left of that line. That percentage depends on both the position of the line (which depends mainly on the latitude) and the shape of the spectrum. The spectral shape has greater scope for longitudinal variation, and we might expect the shape in the most energetic regions to differ from that elsewhere as a result of more dominant non-linear interactions.

In Fig. $8 \mathrm{~b}$ we show for each control point the percentage of the steric power contained at frequencies lower than the Rossby frequency. If our interpretation is right, then this is the percentage of the variance at each point that occurs in the high coherence spectral band, so it should look similar to Fig. 8 a, the percentage of $\phi$ variance explained by a $2.5^{\circ}$ diameter nearby ring. The spectral shape alone introduces the longitudinal structure of Fig. 8a.

\section{Conclusions}

There is geographical variation in the strength of the relationship between island sea level and offshore sea level, as shown in Fig. 3a. This is largely latitudinal and relates to the proportion of the sea-level spectrum that has frequency lower than the local Rossby frequency (Fig. 8).

Although this study was motivated by investigating the sea level at islands, we have found that the decoupling present at this model resolution is not due to the island bathymetry. Thus it is unrelated to the possible mechanisms of wind stress over shallow water or topographic barriers to propagation of density signals, both of which appear to play a part in the decoupling between deep ocean and coastal continental sea level variability seen by Hughes and Williams (2010) and Bingham and Hughes (2012). Instead our control experiment showed that decoupling, similar to that seen at islands, also occurs between deep ocean points and surrounding rings. In that sense, island sea level behaves just like sea level at any open ocean point. This suggests that the decoupling must be related to the spatial scales of the sea level anomalies and their ability to propagate while in deep water.

Our spectral coherence analyses (Figs. 5 and 6) support this conclusion. At high frequencies (periods shorter than about 1 week in the tropics and a few weeks at high latitudes), there is coherence between island and offshore sea level. This is virtually all associated with bottom pressure variability, which typically has large length scales associated with barotropic variability, and dominates the variability out to longer periods at higher latitudes (Vinogradova et al., 2007; Bingham and Hughes, 2008). On moving to lower frequencies, as Fig. 2 shows, steric variability and therefore 
baroclinic processes come to dominate in a spectral region in which such variability cannot propagate as baroclinic Rossby waves. This results in a dip in coherence, but then a rise again, which always occurs at frequencies lower than (to the left of) the yellow line in Fig. 6, representing the maximum frequency at which linear baroclinic Rossby waves can exist. The Rossby wave dispersion relation is such that, immediately to the left of the yellow line, only a narrow range of wavelengths is permitted. The range of permitted wavelengths expands as frequency decreases and, therefore, represents an increasing fraction of the total variability.

Thus, coherent signals appear to be associated with barotropic and wave-like baroclinic processes. The band of low coherence spreads for some (variable) range to either side of the Rossby wave cut-off frequency and appears to represent steric variability, which cannot propagate in a wavelike manner. As noted in Hughes and Williams (2010), although much of the actual variability is nonlinear and may be eddy-like, it still often displays many of the features of baroclinic Rossby waves, including westward propagation resulting from a similar mechanism.

In this interpretation, poor correlation occurs for islands that are surrounded by ocean in which a large fraction of the steric variability is at frequencies and wavelengths for which the baroclinic Rossby wave propagation mechanism does not apply. This situation is more likely to occur at high latitudes, a factor which results in a reduction in coherence as one moves away from the Equator. However, at the highest latitudes the steric signal is not dominant, and coherence due to bottom pressure results in a rise as seen in Fig. 3a.

Other processes will apply to steep and small islands that cannot be resolved by the $1 / 12^{\circ}$ model. However it seems unlikely to us that the correlation between island and off-shore sea level would be increased by such processes, so the pink regions in Fig. 6 represent the maximum extent of the latitudes and periods for which high coherence can be expected.

There appears to be broad agreement between our results and those of Vinogradov and Ponte (2011), although their data are limited to existing gauge locations, so there is a sparser distribution of points, making it harder to see any latitude dependence. The island locations between $20^{\circ} \mathrm{N}$ and $20^{\circ} \mathrm{S}$ have higher correlation between tide gauges and satellite altimetry than those further north (e.g. Canaries, Azores) and south (e.g. Easter Island, San Felix) (see Fig. 6 in their paper). They used time series of annual means, which would explain the slightly higher correlations than in Fig. 3a - our nearest equivalent figure is percentage of variance of island $h$ explained by offshore $h$ filtered to pass periods $>18$ months, which looks very similar (Fig. 3c). They also state that "results based on 2 and $3 \mathrm{yr}$ averages do lead to improved agreement between the tide gauge and [altimetry] records", though with no indication of regional effects.

Vinogradov and Ponte (2011) saw discrepancies between the tide-gauge and altimetry at three islands (San Felix, Juan Fernández and Easter Island) in the south-eastern Pacific, which they ascribed to either vertical land movement or noisy tide-gauge records. Our results also show these islands to have low coherence between on- and off-shore sea level. However, we see that this is consistent with the latitude dependence. Indeed, we find Juan Fernández to have slightly above average coherence for its latitude.

Acknowledgements. Thanks to Rory Bingham for discussions and help with the model diagnostics. We thank an anonymous reviewer for the suggestion of the control experiment. This work was funded by the UK Natural Environment Research Council, using National Capability funding allocated to the National Centre for Earth Observation and the National Oceanography Centre.

Edited by: M. Hecht

\section{References}

Bingham, R. J. and Hughes, C. W.: The relationship between sea-level and bottom pressure variability in an eddy permitting ocean model, Geophys. Res. Lett., 35, L03602, doi:10.1029/2007GL032662, 2008.

Bingham, R. J. and Hughes, C. W.: Local diagnostics to estimate density-induced sea level variations over topography and along coastlines, J. Geophys. Res.-Oceans, 117, C01013, doi:10.1029/2011JC007276, 2012.

Chelton, D., DeSzoeke, R., Schlax, M., El Naggar, K., and Siwertz, N.: Geographical variability of the first baroclinic Rossby radius of deformation, J. Phys. Oceanogr., 28, 433-460, doi:10.1175/1520-0485(1998)028;0433:GVOTFB $i 2.0 . C O ; 2$, 1998.

Emery, W. J. and Thomson, R. E.: Data Analysis Methods in Physical Oceanography, Elsevier, 2001.

Hughes, C. W. and Williams, S. D. P.: The color of sea level: Importance of spatial variations in spectral shape for assessing the significance of trends, J. Geophys. Res.-Oceans, 115, C10048, doi:10.1029/2010JC006102, 2010.

Leuliette, E. W., Nerem, R. S., and Mitchum, G. T.: Calibration of TOPEX/POSEIDON and Jason altimeter data to construct a continuous record of mean sea level change, Mar. Geod., 27, 79-94, doi:10.1080/01490410490465193, 2004.

Marsh, R., de Cuevas, B. A., Coward, A. C., Jacquin, J., Hirschi, J. J. M., Aksenov, Y., Nurser, A. J. G., and Josey, S. A.: Recent changes in the North Atlantic circulation simulated with eddypermitting and eddy-resolving ocean models, Ocean Model., 28, 226-239, doi:10.1016/j.ocemod.2009.02.007, 2009.

Vinogradov, S. V. and Ponte, R. M.: Low-frequency variability in coastal sea level from tide gauges and altimetry, J. Geophys. Res., 116, C07006, doi:10.1029/2011JC007034, 2011.

Vinogradova, N. T., Ponte, R. M., and Stammer, D.: Relation between sea level and bottom pressure and the vertical dependence of oceanic variability, Geophys. Res. Lett., 34, L03608, doi:10.1029/2006GL028588, 2007.

Vignudelli, S., Kostianoy, A., Cipollini, P., and Benveniste, J. (Eds.): Coastal Altimetry, Springer, 1st Edn., 2011. 